\title{
Storytelling corporativo y responsabilidad social corporativa: análisis del caso "The promise" de Volvo
}

Corporate storytelling and corporate social responsability: analysis of the case "The promise" of Volvo

Storytelling corporativo e responsabilidade social corporativa: análise do caso "The promise" da Volvo

Tomás Atarama-Rojas

- Tomás Atarama Rojas es doctorando de Comunicación en la Universidad de los Andes.

- Máster en Creación de Guiones Audiovisuales por la Universidad Internacional de La Rioja (España) y Licenciado en Comunicación por la Universidad de Piura (Perú).

- Actualmente se desempeña como profesor investigador de la Facultad de Comunicación de la Universidad de Piura (Perú), donde imparte las asignaturas de Comunicación Narrativa y Storytelling, y Fundamentos de Guion.

- Su investigación está centrada en el guion cinematográfico y el uso del storytelling en el mundo comercial y corporativo.

- E-mail: tomas.atarama@udep.pe

Carla Sánchez Armas

- Licenciada en Comunicación por la Universidad de Piura.

- Se ha desempeñado como Asistente de Investigación en la Facultad de Comunicación de la Universidad de Piura.

- Actualmente trabaja en el área de Sostenibilidad de Enel Perú, desarrollando la comunicación de la responsabilidad social de la empresa.

- E-mail: sanchezarmascarla@gmail.com 


\section{Resumen}

Este artículo estudia el uso del storytelling dentro de las empresas como herramienta para comunicar eficazmente el compromiso de responsabilidad social corporativa. Se realizará un análisis diegético que se aplicará a la campaña "The promise" de Volvo, en el que se estudiará la evolución del planteamiento, objetivo, trama, conflicto y arco de transformación en la historia de la campaña. De este modo, se identificará la solución que plantea Volvo al conflicto que se narra en la historia, a partir de sus valores corporativos.

PALABRAS CLAVE: STORYTELLING CORPORATIVO •RESPONSABILIDAD SOCIAL CORPORATIVA •VALORES CORPORATIVOS・VOLVO.

\section{Abstract}

This article studies the use of storytelling in corporations as a tool to achieve an effective communication of the corporate social responsibility commitment. This is how we are going to do a diegetic analysis applied to the ad of Volvo called "The promise", in this analysis we will study the evolution of the approach, goal, plot, conflict and the arc of transformation in the story of the ad. In this way, we will identify the solution that poses Volvo to the conflict narrated in the history, based on their corporate values.

KEYWORDS: CORPORATE STORYTELLING • CORPORATE SOCIAL RESPONSIBILITY・CORPORATE VALUES •VOLVO.

\section{Resumo}

Este artigo estuda o uso do storytelling dentro das empresas como ferramenta para comunicar de forma eficaz o compromisso de responsabilidade social empresarial. Será realizada uma análise diegética que será aplicada à campanha "The promise" da Volvo, em que será estudada a evolução da abordagem, do objetivo, da trama, do conflito e do arco de transformação na história da campanha. Desse modo, será identificada a solução que a Volvo propõe para o conflito que se narra na história a partir de seus valores corporativos. 
En una época de convergencia y globalización, que supone grandes esfuerzos de comunicación por parte de las organizaciones, es necesario que la dirección de estas esté orientada a generar una relación con sus públicos internos y externos, bajo un formato que sea capaz de fidelizarlos. Según Costa (2014), es necesario que exista una comunicación corporativa de acuerdo a los nuevos tiempos.

El contexto actual demanda una organización que no solo se enfoque en su productividad y su crecimiento económico, sino que además se centre en generar engagement con sus stakeholders, pues según Gil (2016) son ellos quienes, a través de la responsabilidad social corporativa (RSC), Les otorgan a las empresas ventajas competitivas difíciles de igualar por la competencia. "Es por esto que las empresas buscan trabajar con dos conceptos que se entrelazan: la RSC y la reputación. No se puede generar una reputación corporativa favorable sin un ser, actuar y comunicar socialmente responsable" (Ochoa; Pabón, 2012, p.14).

Ante esta realidad, este artículo pretende estudiar el storytelling corporativo como herramienta eficaz para comunicar el compromiso de RSC. En este sentido, Costa (2014, p. 164) asegura que "el storytelling se ha convertido en una herramienta poderosa para las organizaciones", pues a través de este se cuentan historias por medio de las cuales las empresas se dan a conocer, creando así un lazo emocional con sus stakeholders.

Siguiendo esta línea, Gill (2011) sostiene que las historias son una herramienta versátil, puesto que el narrador puede contar la posición de la organización en base a los intereses de los stakeholders a los que se quiere impactar. Asimismo, Gill (2014) asegura que a menudo el público que recibe la historia de la empresa llega a la misma conclusión que el narrador. En ese sentido, el storytelling ocasiona que las historias puedan transmitir información simbólica de la empresa a sus públicos de interés y, a su vez, lograr que ellos la interpreten de acuerdo al objetivo de la organización. Así, la hipótesis de este trabajo plantea que, por medio del recurso del storytelling corporativo, se logra comunicar el compromiso de RSC a través del modo en que la historia plantea un conflicto que se soluciona a partir de los valores corporativos de la empresa.

Esa hipótesis se busca confirmar en la campaña "The promise" de Volvo, que cuenta una promesa que le hace Volvo al mundo basándose en sus valores corporativos. Volvo narra su compromiso por construir un mundo mejor, por ser una empresa responsable con el medio ambiente y preocupada por la seguridad de sus clientes. A nivel metodológico, se propone aplicar un análisis diegético que estudiará el progreso y la evolución del planteamiento, objetivo, trama, conflicto y arco de transformación en la historia del comercial. Así, en este análisis se identificará la solución que plantea Volvo, por medio del storytelling, y cómo éste ayuda a comunicar efectivamente su compromiso de RSC.

\section{MARCO TEÓRICO}

\section{La RSC como activo empresarial estratégico}

En la actualidad, la preocupación de las empresas es lograr crecimiento económico y, para lograrlo, gestionan una serie de herramientas que les permita maximizar utilidades minimizando costos. Sin embargo, no toman en cuenta que la gestión de la RSC también permite desarrollar tanto a la empresa como a su entorno (Pazos, 2010).

En ese sentido, Ochoa y Pabón (2012) señalan que el contexto actual demanda un nuevo tipo de organización social y económica. La RSC va más allá de una acción social, se ha convertido en un modelo de negocio que busca también obtener un beneficio. Así, para Andreu y Fernández (2011, p. 5), la RSC no solo debería estar vinculada con proyectos sociales', sino que debería

1 En la actualidad, la RSC está más vinculada a los proyectos sociales porque éstos suelen tener alta visibilidad y son fáciles de entender por la opinión pública (Andreu; Fernández, 2011). 
estar relacionada, sobre todo, a gestionar los riesgos sociales, económicos o ambientales derivados del negocio, con el fin de generar un impacto positivo en la sociedad. Por lo tanto, tal y como lo explica Gil (2016), la RSC no tiene por qué verse como un gasto, sino como una ganancia, un nuevo modelo de negocio que hace a la empresa más competitiva, pues hace que ésta emprenda nuevos negocios que son buenos para la corporación y buenos para la sociedad.

Por otro lado, la RSC no solo es capaz de generar rentabilidad, sino que, además, como lo explican Ochoa y Pabón (2012), la RSC da vida a la reputación corporativa, el intangible por el que las empresas trabajan a través del tiempo y el que le otorga competitividad a una organización. Asimismo, gracias a la reputación corporativa, las acciones de RSC tienen un efecto positivo en los resultados económicos de una empresa (Gonzáles; Donate; Guandamillas, 2014).

Según Porter y Kramer (2006), las empresas que desarrollan prácticas de RSC pueden conseguir tres objetivos principales: creación de riqueza, cohesión social y protección del medioambiente. Pero también son capaces de generar recursos intangibles de alto valor estratégico, tales como la reputación corporativa (Gonzáles; Donate; Guandamillas, 2014). Partiendo de esas potencialidades de la RSC, aplicarla al ámbito empresarial resulta totalmente viable y beneficioso, como señalan Porter y Kramer:

[...] si las corporaciones analizaran sus alternativas de responsabilidad social bajo los mismos marcos que orientan sus decisiones de negocios, descubrirían que la RSC puede ser mucho más que un costo, una limitación o un acto de beneficencia; puede ser una fuente de oportunidades, innovación y ventaja competitiva. (Porter; Kramer, 2006, p. 80)

\section{El uso del storytelling para comunicar los valores corporativos de una organización}

Bernasconi, Alfeu y Aleixo (2011) aseguran que el storytelling es un arma poderosa en el ámbito empresarial por el fin persuasivo que posee. Este fin persuasivo tiene un gran calado en las personas y es justamente por eso que las historias pueden ser también usadas como estrategia, herramienta o aliado de comunicación. Es así que Costa (2014, p. 165) define el storytelling corporativo como "el arte (y la técnica) de contar historias al servicio de la identidad de la organización y/o de la marca para dar sentido a su actividad y explicarse a sí misma y a los otros", pues señala que el contar historias es un activo estratégico importante para las organizaciones porque les permite darse a conocer y empatizar con sus públicos internos y externos.

Partiendo de esos atributos del storytelling, podemos afirmar que éste es capaz de comunicar los valores corporativos de una empresa a sus stakeholders. En palabras de Costa (2014, p. 165), el storytelling corporativo es "el camino para que la organización exprese sus valores internos a públicos tanto internos como externos por medio de relatos diferentes (pero coherentes entre sí) que capten su atención, les hagan emocionarse y retener el mensaje". De ese modo, el storytelling se ha convertido en una técnica de comunicación, de control y de poder, puesto que cada vez más organizaciones no gubernamentales (ONG), agencias gubernamentales y grandes empresas descubren su eficacia (Salmon, 2014).

Según Gill (2014), el storytelling corporativo puede aumentar el conocimiento deseado de la organización y dar origen a una cultura empresarial basada en sus valores corporativos y de marca. Tal como señala Salmon (2014, p. 57), "las marcas han perdido su status de objeto o de imágenes 'cosificadas': nos hablan y nos cautivan, nos cuentan historias que tienen que ver con nuestras expectativas y nuestras visiones del mundo".

Es así que, para Bernasconi, Alfeu y Aleixo (2011), el acto de contar historias sobre la organización fortalece la cultura corporativa, pues ésta es fruto de la interacción de los trabajadores de la empresa y es creada, reproducida y mantenida por los públicos. Por ello, aseguran que el storytelling corporativo es más que una herramienta eficaz de alto alcance, es una disciplina de gestión para la comunicación corporativa. 


\section{MATERIAL Y METODOLOGÍA}

\section{Volvo: "The promise"}

El material escogido para poder comprobar la hipótesis de esta investigación es el caso de Volvo, una empresa de origen sueco con 90 años de trayectoria en la industria automotriz, considerada por el Monitor Empresarial de Reputación Corporativa (Merco) dentro del top 50 de las empresas más responsables y con mejor reputación corporativa en el Perú. El ranking internacional que publica anualmente Merco se realiza en distintos países de América y Europa.

Volvo es un ejemplo de cómo una compañía puede sacar provecho del storytelling como herramienta de comunicación a nivel externo. Ese atributo se refleja en su campaña "The promise" (Forsman; Bodenfors, 2016), la cual plantea, de una manera emotiva, una serie de problemas y tragedias que han ocurrido desde que los coches se han masificado a lo largo de los años. Es así que la empresa sueca promete solucionar esos problemas en base a sus valores corporativos y narra su compromiso por construir un mundo mejor, cuidando del medio ambiente y velando por la seguridad de sus clientes. Cabe destacar que existen otras dos piezas audiovisuales que derivan de esa campaña y son de menor duración.

Así, gracias a información encontrada en la web de Volvo, se encontró la historia que la compañía narra a su público externo y su compromiso por ser, cada vez más, una empresa responsable:

\footnotetext{
En Volvo, todo lo que hacemos tiene un mismo principio y un mismo fin: las personas. Por este motivo, el compromiso con un día a día más fácil, más seguro y más cómodo forma ya parte de nuestra forma de ser. Es el ADN de Volvo.

Hoy continuamos tan comprometidos como siempre con nuestros tres grandes valores: la seguridad, la calidad y el respeto por el medio ambiente.

Protegemos lo que realmente importa. Ayudamos a las personas a sentirse especiales. Y contribuimos a convertir el mundo en un lugar mejor para todos.
}

Ahora bien, el criterio para elegir esa campaña es que se han encontrado características fundamentales: (1) pieza audiovisual; (2) pieza publicada en la página web de la empresa; (3) pieza que refleja el compromiso de RSC de la compañía. Además, otro criterio importante para realizar la selección fue el de actualidad, ya que la campaña fue publicada en el 2016.

\section{Metodología}

Este artículo destaca las potencialidades del storytelling corporativo como herramienta de comunicación capaz de dar a conocer a los stakeholders de una compañía, su compromiso de RSC. Así, con el objetivo de estudiar el caso presentado, se realizará un análisis diegético de la campaña "The promise". La forma en la que se estudiará la evolución de la historia seráa través de los distintos elementos que componen el storytelling corporativo cuando lo vinculamos a los valores de la empresa.

El planteamiento es el inicio de la historia: se presenta al protagonista, el tiempo y lugar donde se desarrollan los hechos. Asimismo, se presentan datos importantes sobre el personaje, los cuales se profundizarán durante el desarrollo del relato. Para Atarama-Rojas y Castañeda-Purizaga (2017), eso inicia la acción de la historia. Es así que, para analizar adecuadamente el planteamiento de la historia, se observarán los primeros segundos del comercial principal y se identificará al protagonista y el contexto en el que éste se mueve.

El objetivo, de acuerdo con Atarama-Rojas y Castañeda-Purizaga (2017), es la meta que se plantea el protagonista, la cual mueve las acciones de este y van marcando su crecimiento. Se plantea al inicio de la historia y es esencial para generar 
empatía con la audiencia. Para crear una historia que se vincule con la empresa, es importante materializar los valores y la visión en un objetivo concreto e identificable por la audiencia.

El conflicto, en palabras de Sánchez-Escalonilla (2016, p.19), es un problema por resolver que "se halla en el núcleo de toda historia, de toda unidad dramática y equivale a la lucha, pugna, tensión, dialéctica" presente en toda narración. Asimismo, distingue tres niveles de conflictos: aquel que está en el ámbito de los problemas o tensiones interiores es el conflicto interno; las tensiones generadas entre personajes son los conflictos de relación; los problemas de tipo físico son los conflictos básicos. Para determinar el conflicto en la historia se enumerarán los obstáculos o problemas que se presentan a lo largo de la historia en relación con el objetivo que puede tener la empresa. Asimismo, se analizará cómo esos obstáculos pueden constituirse en un conflicto que represente, a su vez, los problemas que trata de solucionar la compañía a través de sus valores corporativos.

La trama, según Atarama-Rojas y Castañeda-Purizaga (2017), es una sucesión de hechos que ocurren en un determinado tiempo y espacio a lo largo de la historia. La forma y el orden en el que se desarrollan los hechos es transcendental para otorgarle sentido a la historia. De ello, entendemos que la historia que cuenta la empresa debe ser capaz de conectar con su público y despertar su interés a partir de información que aporte credibilidad al relato, además debe transmitir información que suponga un aprendizaje para el público, pues éste debe conocer la labor y el compromiso que tiene la empresa con la sociedad y con sus stakeholders. Así, para analizar adecuadamente la trama se tomará en cuenta el desarrollo de la historia, pues los conflictos van evolucionando junto con ella y se determinará si la trama responde a un conflicto interno, de relación o básico, con el fin de saber de qué manera puede apelar a los valores que representa la empresa.

El arco de transformación, según Brenes (2012), es el movimiento o cambio en el personaje principal que suele darse entre el principio y final de una historia. La personalidad del protagonista va evolucionando a medida que se desarrolle la historia, pues influyen mucho sus acciones y las situaciones que se presentan, así como las experiencias a las que se enfrenta. Es así que, al finalizar el relato, el protagonista no es el mismo, ha sufrido cambios. Para determinar cómo ha evolucionado el protagonista en la historia se estudiará la toma de decisiones del personaje, sus acciones y las situaciones a las que se enfrenta. De esa manera, se podrá analizar cómo ha ido evolucionando el protagonista a lo largo de la historia y cómo éste es al final del relato en relación a la personalidad que tenía al inicio.

\section{RESULTADOS}

\section{Planteamiento y objetivos}

La historia se plantea con el amanecer de una ciudad, en la que se ve a un hombre corriendo, a una mamá despertando a su hija, quien será la narradora, y a una ciudad plagada de pequeñas luces de coches. Luego, se escucha la voz en off de la niña, quien va de pasajera en un automóvil reflexionando sobre lo que los coches solían ser -sinónimo de libertad y diversión-y lo que ahora son -sinónimo de accidentes, muerte y contaminación-, mientras mira a través de la ventana del automóvil accidentes de tránsito y una ciudad llena de smoke.

Volvo, como protagonista de la historia, se enfrenta a una serie de problemas que se le presentan: choques, accidentes de tránsito, contaminación ambiental y la percepción de peligro e inseguridad que las personas tienen sobre los coches. En ese sentido, su objetivo en la historia es solucionar esos problemas creando coches que cuiden y velen por la seguridad de las personas y el medio ambiente y, así, lograr que vuelvan a ser un símbolo de libertad, diversión y seguridad para las personas. 
Eso se corresponde con el compromiso de RSC de Volvo, pues la empresa sueca plantea integrar sus valores corporativos: seguridad, calidad y respeto por el medio ambiente, con la finalidad de solucionar los conflictos a los que se enfrenta la empresa. Por ello, Volvo ha incorporado en sus coches motores Drive-E, un sistema Intellisafe y purificador de aire dentro del automóvil. Además, sus coches están hechos con un 85\% de materiales reciclables.

Esas iniciativas de RSC tienen como objetivo cuidar de la vida de las personas y del mundo en el que viven. Es así que, a partir de esas innovaciones tecnológicas, Volvo muestra su compromiso por reducir el impacto ambiental que generan los automóviles y por velar por la seguridad y la vida de sus conductores y sus seres queridos. De ese modo, los valores corporativos de la empresa sueca se concretizan en sus acciones de RSC.

Esas iniciativas son a su vez un fiel reflejo de la misión de Volvo, pues ésta gira en torno a las personas. Es por ello que fabrican coches con lo último en tecnología para hacer más fácil la vida de las personas, fabrican coches sostenibles para cuidar del medio ambiente, donde habitan las personas, e incorporan a sus vehículos innovaciones tecnológicas de seguridad, porque eso salva la vida de las personas. Así pues, la visión de Volvo para 2020 es que nadie debe morir o resultar gravemente herido en un nuevo automóvil de Volvo.

\section{Conflicto y trama}

En la historia de Volvo se ha identificado la presencia de un conflicto básico. En ese sentido, se ha considerado que los choques, los accidentes de tránsito y la contaminación ambiental constituyen las situaciones que potencialmente pueden impedir que Volvo logre su objetivo. Como se ha podido ver en el planteamiento y objetivos de la historia, Volvo tiene un compromiso con las personas y el mundo en el que viven, por ello que existe entonces un constante riesgo de que problemas, como los mencionados, perjudiquen la misión de Volvo.

Con respecto a la trama de la historia, la cual corresponde a un conflicto básico, se encontró que los constantes peligros que suponen los coches y a los que se encuentran expuestos los conductores y sus seres queridos constituyen eventos realistas para Volvo. En ese sentido, la empresa sueca, a partir de sus valores corporativos, intentará construir un mundo mejor, más seguro y más ecológico.

A nivel de RSC, Volvo enfrenta problemas de contaminación ambiental por los altos niveles de consumo y emisiones que tienen los coches. Asimismo, afronta altos porcentajes de probabilidad de muertes, choques y accidentes a causa de los coches, por lo que, a su vez, enfrenta un riesgo latente y la percepción de peligro e inseguridad que las personas tienen sobre los vehículos.

En ese sentido, en cuanto a la trama de la RSC, Volvo ha incorporado a sus nuevos coches motores Drive- $E$, los cuales tienen un nivel mínimo de consumo y emisiones contaminantes. Asimismo, sus coches están hechos con un $85 \%$ de materiales reciclables. Además, ha incorporado un sistema Intellisafe en sus nuevos coches, el cual está conformado por una serie de innovaciones tecnológicas como alertas de detección de ciclistas, peatones o automóviles, frenos automáticos, jaulas de seguridad, entre otros. Así, la RSC de Volvo busca hacer coches que intenten reducir el número de accidentes, como también cuidar del medio ambiente y velar por la seguridad de las personas.

De ese modo, esas acciones de RSC le incorporan valores a la empresa, en el sentido en que Volvo, a través de sus valores corporativos de seguridad, calidad y respeto por el medio ambiente intenta solucionar un problema de seguridad vial y de transporte con un sentido amplio de cuidado del medio ambiente y la seguridad de los pasajeros. Por ello, la empresa sueca ha lanzado una nueva línea de coches denominada "Volvo 90", que se caracteriza por tener diversas innovaciones tecnológicas, consideradas el mejor paquete del mundo en asistencia al conductor y un sistema integral de seguridad para evitar accidentes. 


\section{Arco de transformación del personaje}

Al inicio de la historia, Volvo plantea a modo de autocrítica una serie de problemas que los coches han ido generando desde que se han masificado a lo largo de todos esos años. Ese personaje se muestra como un agente parte de esos problemas y no como un personaje que contribuye al cambio. Sin embargo, Volvo evoluciona con el desarrollo de la historia y al finalizar el comercial, termina siendo un personaje que contribuye al cuidado de medio ambiente y vela por la seguridad de la familia.

De igual manera, a nivel de RSC, la empresa sueca se plantea reducir el impacto ambiental ocasionado por las emisiones contaminantes de los coches a través de la fabricación de coches sostenibles y el buen aprovechamiento de los recursos naturales. Asimismo, Volvo se plantea cuidar y velar por la seguridad de las personas a través de las innovaciones tecnológicas que han incorporado a sus coches y así cumplir con su promesa: en 2020 nadie sufrirá lesiones graves o mortales en un Volvo nuevo.

Eso se corresponde a su vez con lo que la empresa se plantea, pues, desde 2000, Volvo ha reducido un 50\% el riesgo de sufrir heridas en accidentes con uno de sus vehículos. Es así que, con el fin de cumplir su visión para 2020, Volvo está innovando en la fabricación de coches inteligentes para el futuro, capaces de conducirse solos, con cámaras y radares incorporados que controlarán el tráfico. Esos coches circularán de forma más fluida y eficiente cuidando del medio ambiente y de la seguridad de las personas. En ese sentido, vemos que el nivel de RSC y el nivel de empresa de Volvo es una promesa a la humanidad.

Tabla 1: Análisis diegético de los elementos del storytelling corporativo.

\begin{tabular}{|c|c|c|c|}
\hline Elementos & Historia & RSC & Empresa \\
\hline Planteamiento & $\begin{array}{l}\text { Volvo plantea a modo de } \\
\text { autocrítica una serie de problemas } \\
\text { que los coches han ido generando } \\
\text { desde que se han masificado. }\end{array}$ & $\begin{array}{l}\text { Los valores corporativos son: } \\
\text { seguridad, calidad y respeto } \\
\text { por el medio ambiente. }\end{array}$ & $\begin{array}{l}\text { Las misión de Volvo es } \\
\text { velar por el cuidado y la } \\
\text { seguridad de las personas y } \\
\text { del mundo en el que viven. }\end{array}$ \\
\hline Objetivos & $\begin{array}{l}\text { Solucionar esos problemas } \\
\text { creando coches que cuiden y } \\
\text { velen por la seguridad de las } \\
\text { personas y del medio ambiente. }\end{array}$ & $\begin{array}{l}\text { Cuidar de la vida de las } \\
\text { personas y del mundo } \\
\text { en el que viven. }\end{array}$ & $\begin{array}{l}\text { Su visión es que en } 2020 \\
\text { nadie debe morir o resultar } \\
\text { gravemente herido en un } \\
\text { nuevo automóvil de Volvo. }\end{array}$ \\
\hline Conflicto & $\begin{array}{l}\text { El conflicto básico envuelve: } \\
\text { choques, accidentes de tránsito } \\
\text { y contaminación ambiental. }\end{array}$ & $\begin{array}{l}\text { Altos porcentajes de } \\
\text { probabilidad de muertes, } \\
\text { choques, accidentes } \\
\text { a causa de coches y } \\
\text { contaminación ambiental. }\end{array}$ & $\begin{array}{l}\text { Problemas de seguridad } \\
\text { vial y transporte, así como } \\
\text { el impacto ambiental de las } \\
\text { emisiones de los coches. }\end{array}$ \\
\hline Trama & $\begin{array}{l}\text { Volvo intentará construir } \\
\text { un mundo mejor, más } \\
\text { seguro y más ecológico. }\end{array}$ & $\begin{array}{l}\text { Volvo incorporó a sus nuevos } \\
\text { coches motores Drive- } E \text { un } \\
\text { purificador de aire interno, un } \\
\text { sistema Intellisafey el uso de } \\
\text { materiales reciclables para } \\
\text { la fabricación de sus autos. }\end{array}$ & $\begin{array}{l}\text { Volvo lanzó una nueva línea } \\
\text { de coches denominada } \\
\text { "Volvo 90", que se caracteriza } \\
\text { por sus innovaciones en } \\
\text { asistencia al conductor y } \\
\text { su sistema de seguridad. }\end{array}$ \\
\hline $\begin{array}{c}\text { Arco de } \\
\text { transformación }\end{array}$ & $\begin{array}{l}\text { Volvo contribuye al cuidado } \\
\text { de medio ambiente y vela por } \\
\text { la seguridad de la familia. }\end{array}$ & $\begin{array}{l}\text { Volvo plantea reducir el } \\
\text { impacto ambiental, así } \\
\text { como cuidar y velar por la } \\
\text { seguridad de las personas. }\end{array}$ & $\begin{array}{l}\text { Volvo está innovando en } \\
\text { la fabricación de coches } \\
\text { inteligentes para el futuro. }\end{array}$ \\
\hline
\end{tabular}

Fuente: Elaboración propia (2018). 


\section{DISCUSIÓN Y CONCLUSIONES}

La investigación se planteaba demostrar cómo el recurso del storytelling corporativo logra comunicar el compromiso de la RSC, a través del modo en que la historia plantea un conflicto que se soluciona a partir de los valores de la empresa. Después de haber analizado la campaña de Volvo, se pudo encontrar efectivamente un conflicto claro que la empresa trata de solucionar en el comercial planteando unas líneas de desarrollo, el cual encuentra respaldo en las acciones de RSC de la empresa.

Asimismo, la propuesta de storytelling de la empresa le otorga valor a la organización en la medida que muestra de una manera emotiva y sensible su compromiso de RSC, con lo que logra empatizar con el público. Eso es coherente con lo que Gill (2011), López-Hermida e Ibieta (2013), Costa (2014) y Atarama-Rojas y Castañeda-Purizaga (2017) sostienen, pues esos autores aseguran que la clave para lograr el éxito en una narración es conectar emocionalmente con el público al que se dirige. Lo que efectivamente está haciendo la empresa.

Para lograr esa propuesta emotiva, la trama de la historia tuvo un rol fundamental, pues según la propuesta de Kent (2015), que enuncia 20 tramas maestras, podríamos decir que Volvo conjuga las tramas de búsqueda, pues se plantea construir un mundo mejor para las personas; y descubrimiento, porque Volvo se plantea a modo de autocrítica una serie de problemas que han ocurrido desde que los coches se han masificado.

En el caso de Volvo, se puede apreciar que la RSC se entiende como el buen hacer de la actividad a la que se dedica la empresa. De acuerdo con Andreu y Fernández (2011), la RSC no sólo debe estar vinculada a campañas sociales, sino que debería estar orientada a brindar soluciones y a gestionar los riesgos derivados del negocio en aspectos sociales, económicos o ambientales, para así generar un impacto positivo en la sociedad en la cual opera.

Un punto relevante en esta investigación, que saltó a la vista a partir del análisis de la campaña y que puede abrir una línea de debate, es la línea transversal que se ha cruzado entre empresa, RSC y storytelling, pues se han traído elementos de la escritura de ficción audiovisual al mundo corporativo. Así, ahora no sólo nos damos cuenta que el storytelling es una herramienta que logra comunicar los objetivos de una empresa, sino que además logra aunar valores a la empresa a partir del compromiso de RSC.

\section{REFERENCIAS}

ANDREU, Alberto; FERNÁNDEZ, José Luis. De la RSC a la sostenibilidad corporativa: una evolución necesaria para la creación de valor. Harvard Deusto Business Review, Barcelona, n. 207, p. 5-21, 2011. Disponível em: <https://goo.gl/6D3r34>. Acesso em: 15 maio 2017.

ATARAMA ROJAS, Tomás; CASTAÑEDA-PURIZAGA, Lucía. La ruptura de la rutina y la soledad de los protagonistas como detonante de las grandes historias breves: análisis de los cortometrajes animados ganadores del Oscar (2011-2015). Revista de Comunicación, Piura, v. 16, n. 1, p. 9-28, 2017. D0I: 10.26441/RC16.1-2017-A1.

BERNASCONI, Analú; ALFEU, Adenil; ALEIXO, Dalva. Storytelling empresarial: relações públicas contador de histórias. In: CONGRESSO DE CIÊNCIAS DA COMUNICAÇÃO NA REGIÃO SUDESTE, 16., 12 a 14 de maio de 2011, São Paulo. São Paulo: Intercom, 2011. Disponível em: <https://goo.gl/1ZaQjf>. Acesso em: 20 mar. 2017. 
BRENES, Carmen. Buenos y malos personajes. Una diferencia poética antes que ética. Revista de Comunicación, Piura, n. 11, p. 7-23, 2012. Disponível em: <https://goo.gl/1iasfq>. Acesso em: 30 jun. 2017.

COSTA-SÁNCHEZ, Carmen. Storytelling y audiovisualización de la comunicación corporativa: las claves de la campaña "Gracias por elegirnos" (Balay). Organicom - Revista Brasilera de Comunicación Organizacional y Relaciones Públicas, São Paulo, v. 11, n. 20, p. 162-176, 2014. Disponível em: <https://goo.gl/nGYDCT>. Acesso em: 20 mar. 2017.

GIL, Guacimara. Análisis discursivo del relato empresarial sobre responsabilidad social corporativa: Coordenadas narrativas y prácticas discursivas. Recerca, Castellón, n. 20, p. 127-156, 2016. D0I: 10.6035/Recerca.2017.20.7.

GILL, Rob. An integrative review of storytelling: Using corporate stories to strengthen employee engagement and internal and external reputation. PRism, Melbourne, v. 8, n. 1, p. 1-16, 2011. Disponível em: <https://goo.gl/t7RpTo>. Acesso em: 20 mar. 2017.

GILL, Robert. Why the PR strategy of storytelling improves employee and ads value to CSR: An integrated literature review. Public Relations Review, Amsterdam, v. 41, n. 5, p. 662-674, 2014. D0I: 10.1016/j.pubrev.2014.02.012.

GONZÁLES, María; DONATE, Mario; GUADAMILLAS, Fátima. El efecto del papel mediador de la reputación corporativa en la relación entre la RSC y los resultados económicos. Revista de Estudios Empresariales - Segunda época, Jaén, n. 1, p. 67-89, 2014. Disponível em: <https://goo.gl/8VM4hR>. Acesso em: 25 maio 2017.

KENT, Michael. The power of storytelling in public relations: Introducing the 20 master plots. Public Relations Review, Amsterdam, n. 41, p. 480-489, 2015. D0I: 10.1016/j.pubrev.2015.05.011.

LÓPEZ-HERMIDA, Alberto; IBIETA, Nicolás. Nuevas narrativas en Comunicación de Salud: El storytelling y la conquista emocional del paciente. Disertaciones: Anuario electrónico de estudios en Comunicación Social, Bogotá, v. 6, n. 2, p. 47-71, 2013. Disponível em: <https://goo.gl/mmKiX2>. Acesso em: 29 maio 2017.

OCHOA, Daniela; PABÓN, María. La reputación corporativa: la gestión del capital relacional de la organización. Comunicación, Medellín, n. 29, p. 13-18, 2012. Disponível em: <https://goo.gl/3gjgyj>. Acesso em: 20 mar. 2017.

PAZOS, Arturo. Gestión de la responsabilidad social empresarial. Cultura, Lima, n. 24, p. 1-20, 2010. Disponível em: <https:// goo.gl/AhLGXm>. Acesso em: 25 maio 2017.

PORTER, Michael; KRAMER, Mark. Estrategia y sociedad: el vínculo entre ventaja competitiva y responsabilidad social corporativa. Harvard Business Review, Brighton, v. 84, n. 12, p. 78-92, 2006.

SALMON, Christian. Storytelling: la máquina de fabricar historias y formatear mentes. Barcelona: Ediciones Península, 2008.

SÁNCHEZ-ESCALONILLA, Antonio. Del guion a la pantalla: lenguaje visual para guionistas y directores de cine. Barcelona: Ariel, 2016.

THE PROMISE. Volvo Cars. Forsman \& Bodenfors. 2016. Disponível em: <https://www.fb.se/work/volvo/the-promise>. Acesso em: 20 mar. 2017.

VIZCAINO, Pablo. Del storytelling al storytelling publicitario. el papel de las marcas como contadoras de historias. 2016. Tese (Doutorado em Comunicação Audiovisual) - Universidade Carlos III de Madrid, Madrid, 2016.

Artículo recibido el 28.07.2017 y aprobado el 30.10.2017. 